\title{
Hysteresis properties of a mixed ferromagnetic-ferrimagnetic ternary alloy
}

\author{
${ }^{1}$ Zeynep DEMIR VATANSEVER(0000-0002-7726-5168) \\ ${ }^{*}$ Department of Physics, Faculty of Science, Dokuz Eylul University, İzmir, Turkey \\ zeynep.demir@deu.edu.tr
}

Arrival Date: 21.10.2017 Accepted Date: 31.05.2018

\begin{abstract}
:
In this work, we have investigated the hysteresis properties of a mixed ferro-ferrimagnetic ternary alloy system by Monte Carlo simulation method. The ternary alloy system contains two interpenetrating sublattices. One of the sublattice consists of type-A magnetic components with spin-3/2 while the other one is randomly occupied type-B and type-C magnetic components with spin-1 and spin-5/2, respectively. We consider both ferromagnetic and antiferromagnetic exchange interactions between nearest-neighbor magnetic components. The influences of the exchange interaction ratio, $\mathrm{R}$, and the concentration value of type- $\mathrm{B}$ magnetic ions, $\mathrm{p}$, on the remanent magnetization, coercivity and hysteresis loops of the system have been investigated in detail. It has been demonstrated that it is possible to modify the remanent magnetization, coercivity and also the saturation magnetization of the system by changing the concentration value and exchange interaction ratio.
\end{abstract}

Keywords: Hysteresis, ternary alloy, Monte Carlo simulation

\section{INTRODUCTION}

Molecule-based magnets are center of interest during the last two decades thanks to their superior properties as compared to metal or metal-oxide magnets which make them attractive for technological applications [1]. Especially, a particular type of molecule based magnets, the multi-metal Prussian blue analogs, are in active investigations since the pioneering works of Okhoshi and his co-workers [2, 3, 4]. They have designed ternary metal Prussian blue compounds of the type $\left(\boldsymbol{N i} \boldsymbol{i}_{\boldsymbol{p}}^{I I} \boldsymbol{M n}_{1-p}^{I I}\right)_{1.5} \boldsymbol{C r}^{I I I}(\mathrm{CN})_{\mathbf{6}}$ which incorporate both ferromagnetic $\left(\boldsymbol{J}_{\boldsymbol{N i}-\boldsymbol{C r}}>\mathbf{0}\right)$ and antiferromagnetic $\left(\boldsymbol{J}_{\boldsymbol{M n}-\boldsymbol{C r}}<\mathbf{0}\right)$ superexchange interactions. The magnetic properties of these ternary metal Prussian blue compounds such as the saturation magnetization, Weiss temperature and coercive field can be well controlled by varying the active concentration of the magnetic ions in the compound [2]. The multi-metal Prussian blue analogs are also shown to display a photo induced magnetic pole inversion [5], two compensation temperatures [6, 7] and inverted magnetic hysteresis loop [8]. Moreover, nano-structured magnets comprised of Prussian blue analogs for instance, nanoparticles [9, 10,11], nanowire arrays [12, 13] and nanotubes [14] have been synthesized in recent years.
On the theoretical side, magnetic properties of ternary Prussian blue analogs of the type $A B_{p} C_{1-p}$ are investigated by several methods such as mean field theory (MFT) $[15,16,17,18,19,20$, 21, 22], effective field theory (EFT) [23, 24, 25], Bethe lattice approximation [26, 27], Green's function technique [28], Monte Carlo (MC) simulation method [29, 30, 31, 32, 33, 34, 35, 36]. In these studies, in accordance with real mixed ferro-ferrimagnets which comprised of Prussian blue analogs [2], the ternary alloy is considered to be composed of two sublattices. One of the sublattice contains type-A magnetic components which interact ferromagnetically $\left(J_{A B}>0\right)$ and antiferromagnetically $\left(J_{A C}<0\right)$ with type-B and type- $C$ magnetic components, respectively. Also, type-B and type-C magnetic components are distributed randomly throughout the other sublattice with probabilities p and 1 - p, respectively.

One of the interesting feature that has been observed in some of these theoretical studies is the existence of a special interaction ratio $R_{C}=$ $\left|J_{A C}\right| / J_{A B}$ where the critical temperature of the system becomes independent of the concentration ratio $p$ of the ternary alloy. For instance in Ref. [31], the authors have determined the critical interaction ratio, $R_{C}$, for different lattice stoichiometries in the case of a three-dimensional Ising system with MC simulations. They have 
showed that $R_{C}$ value changes very little with the lattice stoichiometry and lattice dimension. Also, many of the studies have focused on compensation temperatures of mixed ferro-ferrimagnetic ternary alloys which have been observed for certain values of the Hamiltonian parameters [30, 31, 35].

Despite the extensive theoretical studies about the magnetic properties of ternary alloys, to the best of our knowledge, a detailed study about hysteresis features of these systems has not been reported yet. Thus, our objective in this work is to present a detailed investigation of the hysteresis properties of the mixed ferro-ferrimagnetic ternary alloy of the type $A B_{p} C_{1-p}$ by means of Monte Carlo simulation based on Metropolis algorithm. The ternary alloy system includes three types of magnetic components A with spin-3/2, B with spin-1 and C with spin-5/2. We have examined the effects of the mixing ratio $\mathrm{p}$ and also the exchange interaction ratio $\mathrm{R}$ on the hysteresis loops, coercive field and remanent magnetization of the ternary alloy for a wide range of temperature values. We believe that this study will shed light on the future experimental and theoretical investigations of mixed ferroferrimagnetic compounds in the presence of an external magnetic field.

The paper is organized as follows. In section 2, the details of the theoretical model and our MC simulations are given. Our numerical results are presented in section 3. Finally, our conclusions are summarized in section 4 .

\section{MODEL AND METHOD}

We consider a ternary alloy system of the type $A B_{p} C_{1-p}$ which contains two interpenetrating sublattices. One of the sublattice is occupied by type-A magnetic components while each site of the second sublattice is randomly occupied by type-B and type-C magnetic components with probabilities $\mathrm{p}$ and $1-\mathrm{p}$, respectively (see Figure 1). Thus, the Hamiltonian of ternary alloy system can be written as:

$$
\mathrm{H}=-J_{A B} \sum_{<i j>} S_{i}^{A} S_{j}^{B} \delta_{j}-J_{A C} \sum_{<i j>} S_{i}^{A} S_{j}^{C}\left(1-\delta_{j}\right)-H \sum_{i} S_{i}^{A}-H \sum_{j}\left[S_{j}^{B} \delta_{j}+S_{j}^{C}\left(1-\delta_{j}\right)\right]
$$

Here, $\boldsymbol{\delta}_{\boldsymbol{j}}$ is a random variable which is unity (zero) if site- $\mathrm{j}$ is occupied by type-B (type-C) magnetic atoms. The first and second summations are over the pairs of the nearest-neighbor sites. $\boldsymbol{S}^{\boldsymbol{A}}, \boldsymbol{S}^{\boldsymbol{B}}$ and $\boldsymbol{S}^{C}$ are Ising spin variables which take the values of $S^{A}= \pm 1 / 2, \pm 3 / 2, \quad S^{B}=0, \pm 1$ and $S^{C}=$ $\pm 1 / 2, \pm 3 / 2, \pm 5 / 2$ for type-A, type-B and type-C magnetic components, respectively. The choice of the spin magnitudes correspond to the ternary metal Prussian blue compound, $\left(N i_{p}^{I I} \mathrm{Mn}_{1-p}^{I I}\right)_{1.5} \mathrm{Cr}^{I I I}(C N)_{6}$, where $\mathrm{A}=\mathrm{Cr}$ with $S^{C r}=3 / 2, \mathrm{~B}=\mathrm{Ni}$ with $S^{N i}=1$ and C=Mn with $S^{M n}=5 / 2 \quad$ [3]. Also, the exchange interaction between the components are reported as $\mathrm{J}_{\mathrm{Ni}-\mathrm{Cr}}>$ 0 and $J_{M n-C r}<0$ [3]. Thus, we assume that there exists ferromagnetic interaction between type-A and type-B magnetic components $\left(J_{A B}>0\right)$ whereas type-A and type- $C$ components interact antiferromagnetically $\left(J_{A C}<0\right)$.

We have investigated the hysteresis features of the ternary alloy system by MC simulation method with single spin-flip Metropolis algorithm [37, 38]. As shown in Figure 1, ternary alloy system is located on a square lattice with $\mathrm{L}=128$ and we apply periodic boundary conditions in all directions. The simulations are started at high

$$
M_{A}=\left[\left\langle m_{A}\right\rangle\right]=\left[\frac{1}{N_{A}}\left\langle\sum_{i=1}^{N_{A}} S_{i}^{A}\right\rangle\right]
$$

temperature from $\boldsymbol{k}_{\boldsymbol{B}} \boldsymbol{T} / \boldsymbol{J}_{\boldsymbol{A} \boldsymbol{B}}=7.0$ with randomly oriented spins and the system is gradually cooled with a temperature step of $\boldsymbol{k}_{\boldsymbol{B}} \boldsymbol{\Delta T} / \boldsymbol{J}_{\boldsymbol{A} \boldsymbol{B}}=0.1$ where $\boldsymbol{k}_{\boldsymbol{B}}$ and $\boldsymbol{T}$ are Boltzmann constant and absolute temperature, respectively. $\mathbf{5} \mathbf{1 0}^{\mathbf{4}}$ MC steps per site (MCSS) are used for calculation of the thermal averages of interested physical quantities after discarding the first $\mathbf{1 0}^{4}$ MCSS in order to obtain the equilibrium state. When the configuration for the interested temperature value is obtained, then magnetic field from $\boldsymbol{H} / \boldsymbol{J}_{\boldsymbol{A B}}$ to $-\boldsymbol{H} / \boldsymbol{J}_{\boldsymbol{A} \boldsymbol{B}}$ (decreasing field branch of the hysteresis) is applied. The configuration obtained for $-\boldsymbol{H} / \boldsymbol{J}_{\boldsymbol{A} \boldsymbol{B}}$ is taken as initial configuration for the increasing field branch and in a similar manner, by applying magnetic field from $-\boldsymbol{H} / \boldsymbol{J}_{\boldsymbol{A B}}$ to $\boldsymbol{H} / \boldsymbol{J}_{\boldsymbol{A B}}$, increasing field branch is completed. At each magnetic field step, the first $\mathbf{5} \mathbf{1 0}^{\mathbf{3}}$ MCSS are excluded for thermalization and the magnetizations are calculated over the next $5 \mathbf{1 0}^{3}$ MCSS. We have performed 20 independent computer experiments which correspond to different initial states.

We have calculated the thermal average and configurational average of sublattice magnetizations $\left(\boldsymbol{m}_{\boldsymbol{A}}, \boldsymbol{m}_{\boldsymbol{B}}, \boldsymbol{m}_{\boldsymbol{c}}\right)$ per spin:

$M_{B}=\left[\left\langle m_{B}\right\rangle\right]=\left[\frac{1}{N_{B}}\left\langle\sum_{i=1}^{N_{B}} S_{i}^{B}\right\rangle\right]$ 


$$
M_{C}=\left[\left\langle m_{C}\right\rangle\right]=\left[\frac{1}{N_{C}}\left\langle\sum_{i=1}^{N_{C}} S_{i}^{C}\right\rangle\right]
$$

and total magnetization, $m_{T}$ :

$$
M_{T}=\left[\left\langle m_{T}\right\rangle\right]=\left[\frac{1}{N_{T}}\left\langle m_{A}+m_{B}+m_{C}\right\rangle\right]
$$

Here, $\langle\ldots\rangle$ denotes the thermal averages and [...] corresponds to the configurational averages. In order to determine the phase transition point separating the ordered and disordered phases from each other, we consider the thermal variation of susceptibility defined by:

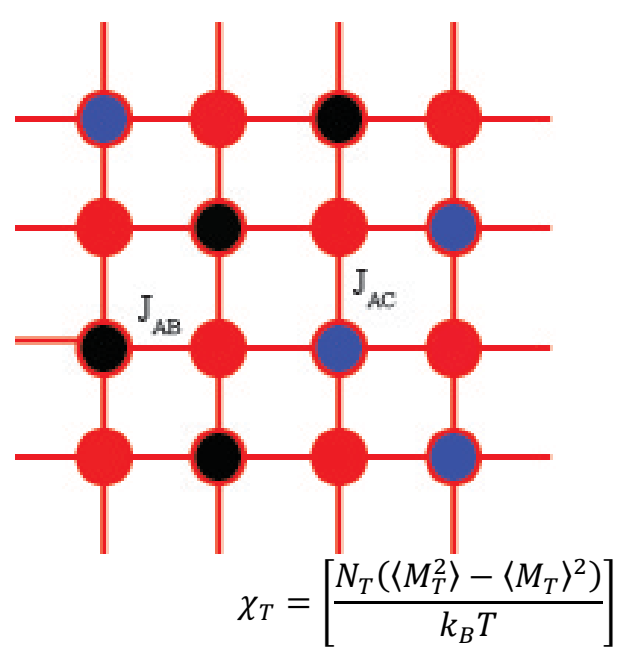
the

total

$$
\chi_{T}=\left[\frac{N_{T}\left(\left\langle M_{T}^{2}\right\rangle-\left\langle M_{T}\right\rangle^{2}\right)}{k_{B} T}\right]
$$

Type - A

Type-B

Type - C

Figure 1: Schematic representation of the ternary alloy system. The red, black and blue circles correspond to type-A, type-B and type-C magnetic components, respectively.

\section{RESULTS}
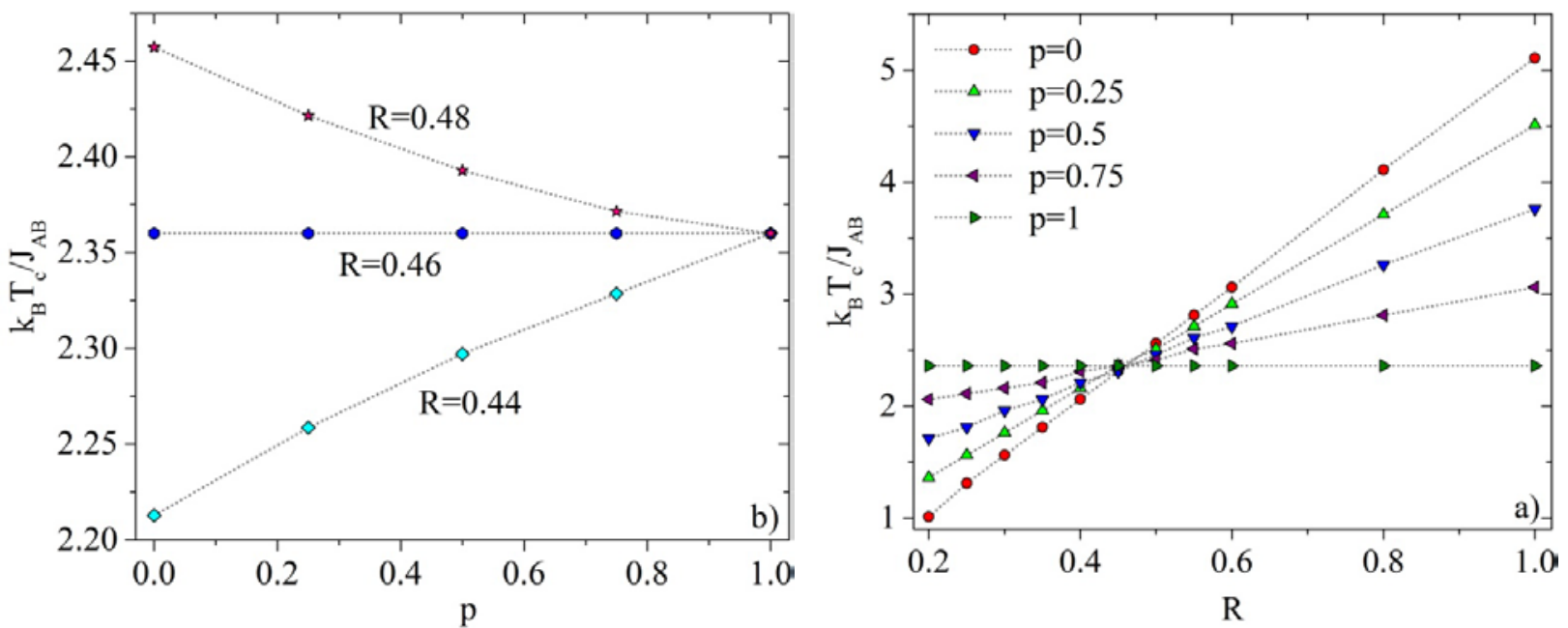

Figure 2: Magnetic phase diagrams of the ternary alloy system in a) $\left(R-k_{B} T_{C} / J_{A B}\right)$, b) $\left(p-k_{B} T_{C} / J_{A B}\right)$ plane.

In this section, we will discuss the effects of an external cycling magnetic field on the magnetic properties of a ternary alloy of the type $A B_{p} C_{1-p}$. Particularly, we pay attention to the effects of the exchange interaction ratio, $R=\left|J_{A C}\right| / J_{A B}$, and mixing ratio, $p$, on the magnetic properties of the system. Obviously, $\mathrm{p}=0$ case corresponds to a ferrimagnetic mixed spin-3/2 and spin-5/2 system while for $p=1$, we have a mixed spin-3/2 and spin-1 ferromagnetic system. For the latter case, there are no $J_{A C}$ exchange interaction terms in the system which means that magnetic properties of the system does not alter with $p$. Also, starting 
from small $R$ values, as $R$ takes larger values, the antiferromagnetic exchange interaction between type-A and type- $\mathrm{C}$ magnetic components becomes effective in the system.

Firstly, in order to confirm the accuracy of our results and also to determine the temperature and concentration values to be studied, we show the phase diagram of the ternary alloy in $(R-$ $\left.k_{B} T_{C} / J_{A B}\right)$ and $\left(p-k_{B} T_{C} / J_{A B}\right)$ planes in Figure 2(a) and Figure 2(b), respectively. As one can readily see from Figure 2(a) that the phase separation curves belonging to different $p$ values intersect at a special interaction ratio, $R_{C}$ for which the transition temperature of the system does not vary with $p$. We have also performed further simulations in a narrow range of $R$ in order to determine accurate value of $R_{C}$ as shown in Figure 2(b). According to our simulation results for the case of a two dimensional ternary alloy system, the special interaction ratio is $R_{C}=0.46$. For $\mathrm{R}<R_{C}$ $\left(R>R_{C}\right)$, the transition temperature of the system increases (decreases) as p takes larger (smaller) values. These results are in accordance with the previous investigations of ternary alloy systems reported in literature [29, 36].
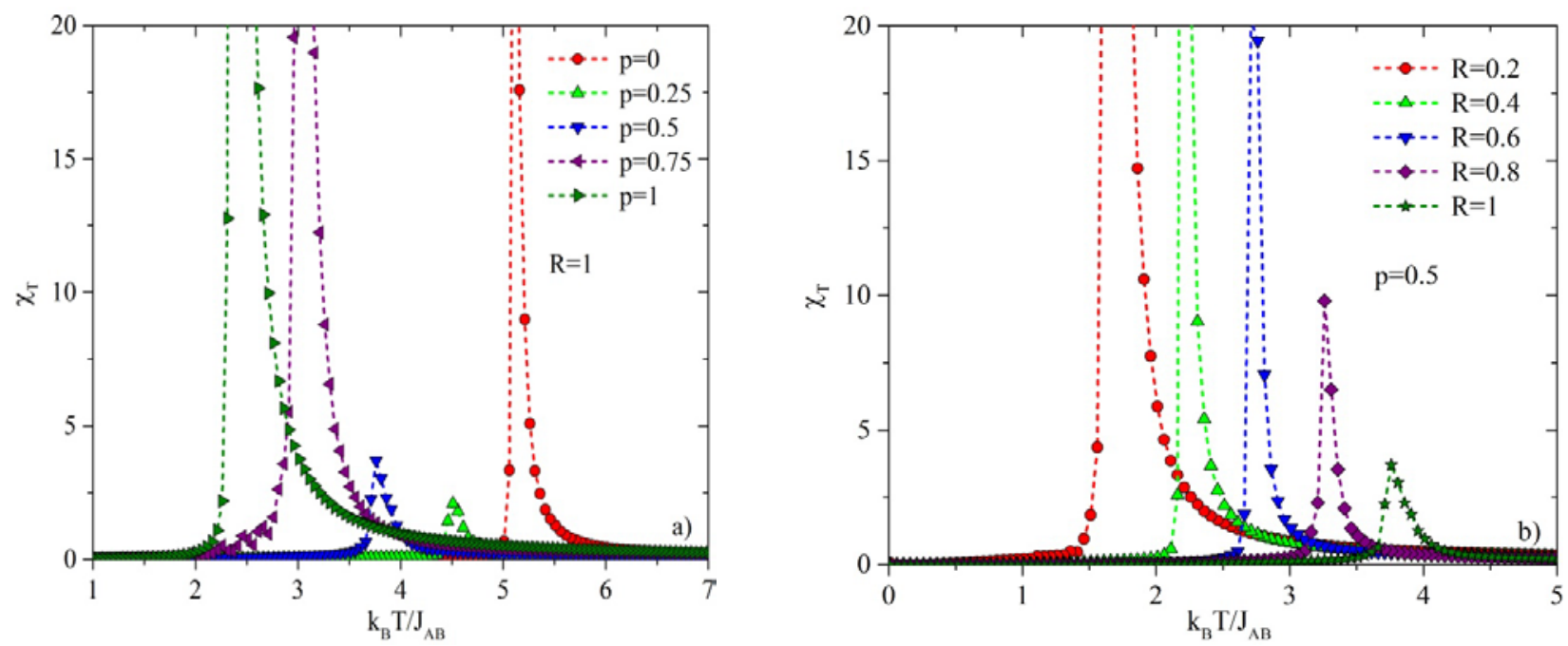

Figure 3: Total susceptibility per spin as a function of temperature a) for $\mathrm{R}=1$ with several values of concentration value of type-B atoms: $\mathrm{p}=0,0.25,0.5,0.75$ and $1 \mathrm{~b}$ ) for $\mathrm{p}=0.5$ with varying values of the interaction parameter: $\mathrm{R}=0.2,0.4,0.6,0.8$ and 1 .

Total susceptibility curves of the system as a function of temperature are shown in Figure 3(a) for $\mathrm{R}=1$ with several values of concentration of type-B atoms. In accordance with the expectations, the susceptibility curves exhibit a maximum at the transition temperature of the system and this maximum moves to higher temperature region with decreasing p. In order to see the effects of interaction parameter, $\mathrm{R}$, on the transition temperature of the system, we also show the total susceptibility curves in the case of concentration of $\mathrm{p}=0.5$ for varying values of $\mathrm{R}$ in Figure 3(b). As one can readily see, as the interaction parameter takes larger values, susceptibility exhibits a maximum at higher temperature region.

These findings also support the phase diagram of the system (Fig 2(a)). In Figure 4(a)-(b), coercive field $H_{c}$, and remanent magnetization, $M_{r}$, of the ternary alloy as a function of $R$ for several values of concentration value, $p$ at a reduced temperature of $k_{B} T_{C} / J_{A B}=1.0$ have been displayed. The coercivity of the ternary alloy system shows an increment behavior as the exchange interaction ratio takes larger values for all $p$ values under consideration. Since the number of $J_{A C}$ spin-spin coupling terms increases with the number of typeC magnetic components in the system, the variation of $H_{c}$ with $R$ is more pronounced in the case of small $p$ values. It can be also seen from the figure that for a fixed value of $R$, the coercivity and remanence strongly depend on the concentration value of type-B magnetic components in the system. Moreover, for all the $\mathrm{p}$ values except for $p=0$ and $p=1$ the remanent magnetization firstly decreases slightly with $R$ because of the enhanced antiferromagnetic exchange interaction in the system whereas an opposite is situation is true for $p=0$. 
With a further increment in $R$, remanent magnetization becomes very close to a value of $M_{T}=\frac{1}{2}\left[\mid M_{A}(T=\right.$ $\left.0)|+p| M_{B}(T=0)|-(1-p)| M_{C}(T=0) \mid\right]$ for all the considered values of $p$.
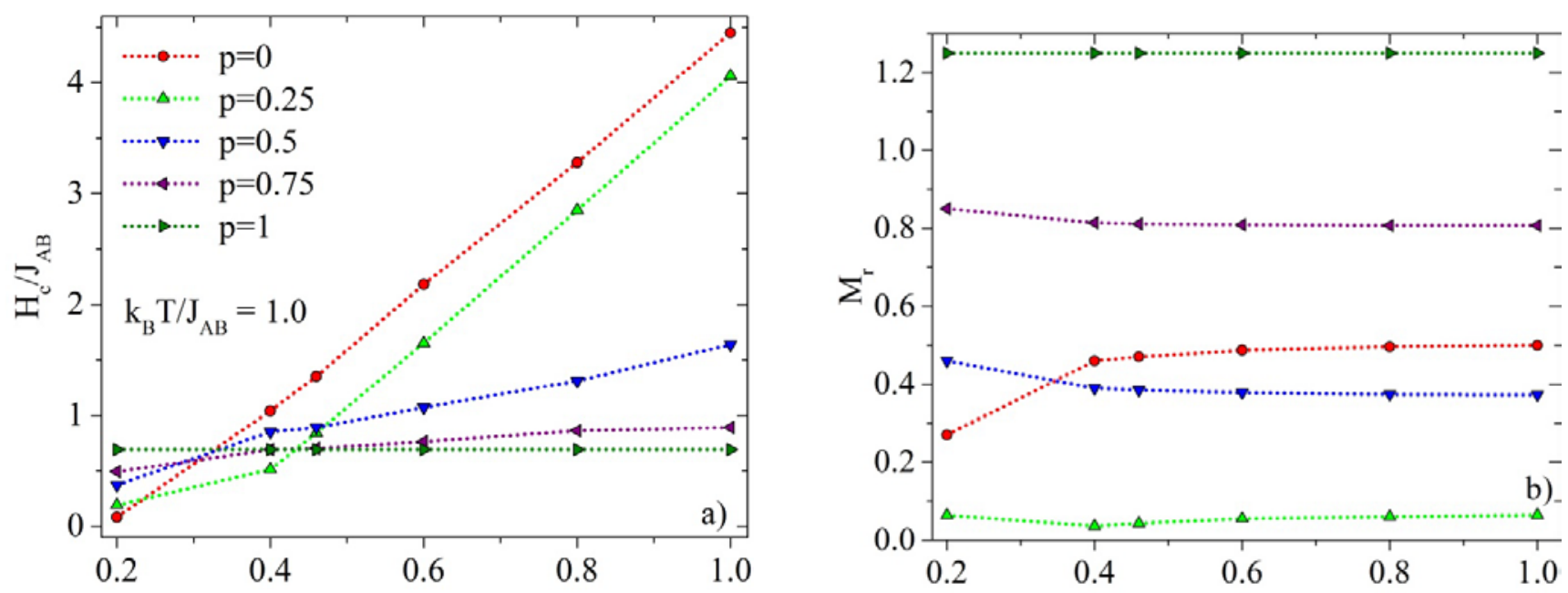

Figure 4: a) Coercivity and b) remanence variations of the ternary alloy system as a function of $R$ at $k_{B} T_{C} / J_{A B}=1.0$ for several values of concentration value of type-B atoms: $p=0,0.25,0.5,0.75$ and 1 .
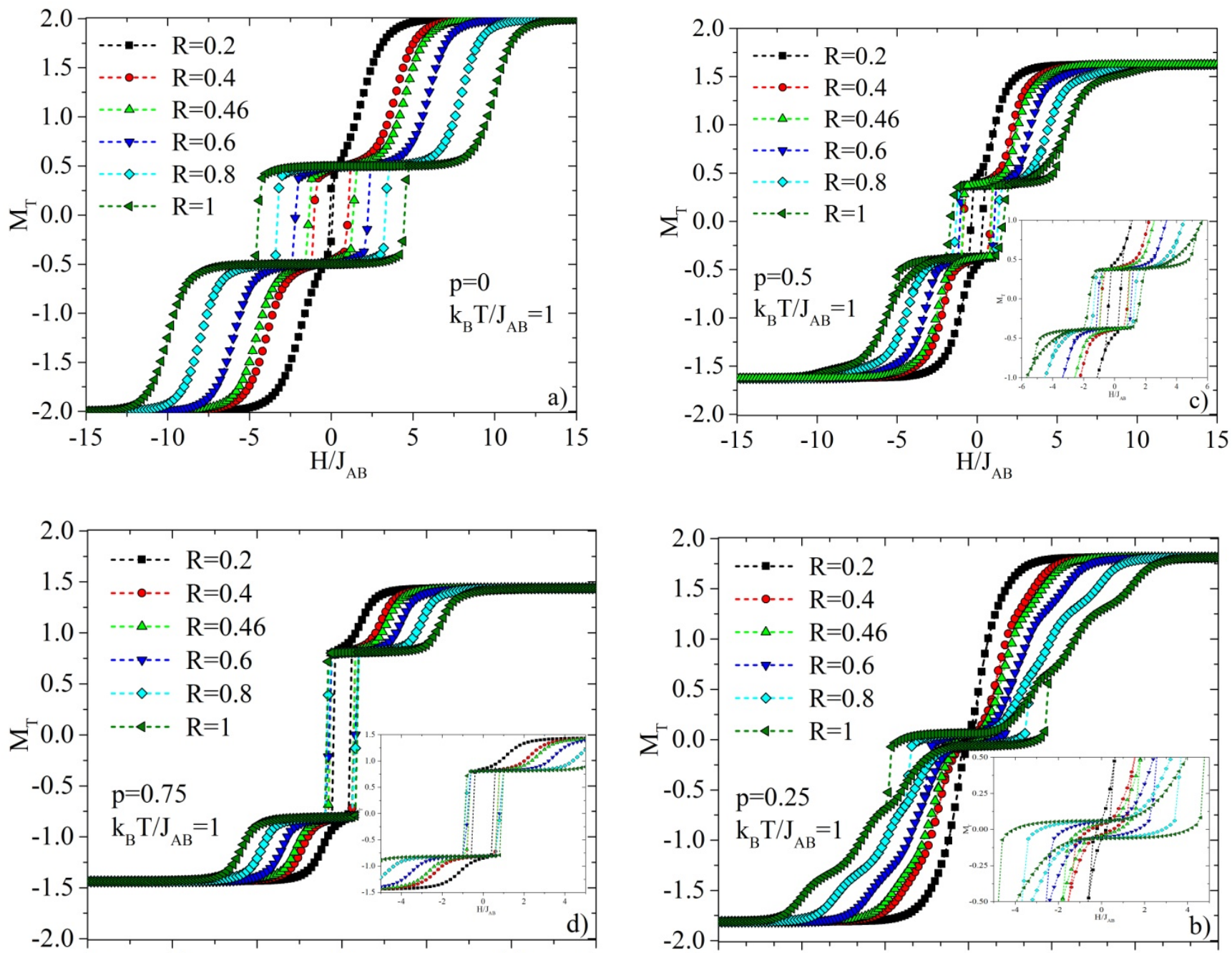

Figure 5: Hysteresis curves of the ternary alloy with a) $p=0$, b) $p=0.25$, c) $p=0.5$, d) $p=0.75$ for several value of R. The curves are shown for reduced temperature value of $k_{B} T_{C} / J_{A B}=1$. 
In order to investigate magnetic features of the ternary alloy, we have showed the hysteresis curves of the system in the case of varying values of $\mathrm{R}$ for a) $\boldsymbol{p}=\mathbf{0}$, b) $\boldsymbol{p}=\mathbf{0 . 2 5}$, c) $\boldsymbol{p}=\mathbf{0 . 5}$, d) $\boldsymbol{p}=\mathbf{0 . 7 5}$ at a reduced temperature of $\boldsymbol{k}_{B} \boldsymbol{T}_{C} / \boldsymbol{J}_{A B}=$ 1.0 in Figure 5. As one can also see from the phase plane of Figure 2(a), the system is in the ordered phase for all $\boldsymbol{p}$ values at this temperature value. While the hysteresis curves display various shapes depending on the value of the concentration value of type-B magnetic components, they have some common features. For instance, if the magnetic field is large enough, it can overcome the antiferromagnetic exchange coupling. Therefore magnetization value saturates to a value $\boldsymbol{M}_{T}=$ $\frac{1}{2}\left[\left|M_{A}(T=0)\right|+p\left|M_{B}(T=0)\right|+(1-\right.$

$\left.\boldsymbol{p})\left|\boldsymbol{M}_{\boldsymbol{C}}(\boldsymbol{T}=\mathbf{0})\right|\right]$ corresponding to the case for which all the spins are aligned parallel to each other and to the external field. Also, starting from increasing or decreasing field branch, as the absolute value of the magnetic field decreases and reaches a particular value (depending on $\boldsymbol{p}$ ), the antiferromagnetic exchange coupling becomes effective and magnetization decreases to the value of $M_{T}=\frac{1}{2}\left[\left|M_{A}(T=0)\right|+p\left|M_{B}(T=0)\right|-(1-\right.$ $\left.\boldsymbol{p})\left|\boldsymbol{M}_{\boldsymbol{C}}(\boldsymbol{T}=\mathbf{0})\right|\right]$. We should also notice that as $\boldsymbol{R}$ takes larger values, the hysteresis loops become wider in accordance with our observations of Figure 4. Another interesting point that should be underlined is the existence of triple hysteresis loops for $\boldsymbol{p}=\mathbf{0 . 5}$ at particular values of exchange interaction ratio. This can explicitly be seen from the inset of Figure 5(c). A triple hysteresis loop is observed for $\boldsymbol{R}=\mathbf{0 . 7 5}$ and $\boldsymbol{R}=\mathbf{1}$ and below a particular of $\boldsymbol{R}$ single hysteresis loop is observed.

In Figure 6(a) and 6(b), the variation of the hysteresis loops with $\mathrm{p}$ is illustrated at $k_{B} T_{C} / J_{A B}=$ 1.0 for $R=0.2$ and $R=1$ respectively. If one compares the width of the hysteresis loops, the hysteresis loops become narrower (wider) for $R=1(R=0.2)$ with increasing $p$. The alteration of remanent magnetization with $\mathrm{p}$ is also in agreement with our discussions for Figure 4(b). Besides, the absolute value of the total magnetization at large fields increases with increasing number of type-C magnetic components in the system.
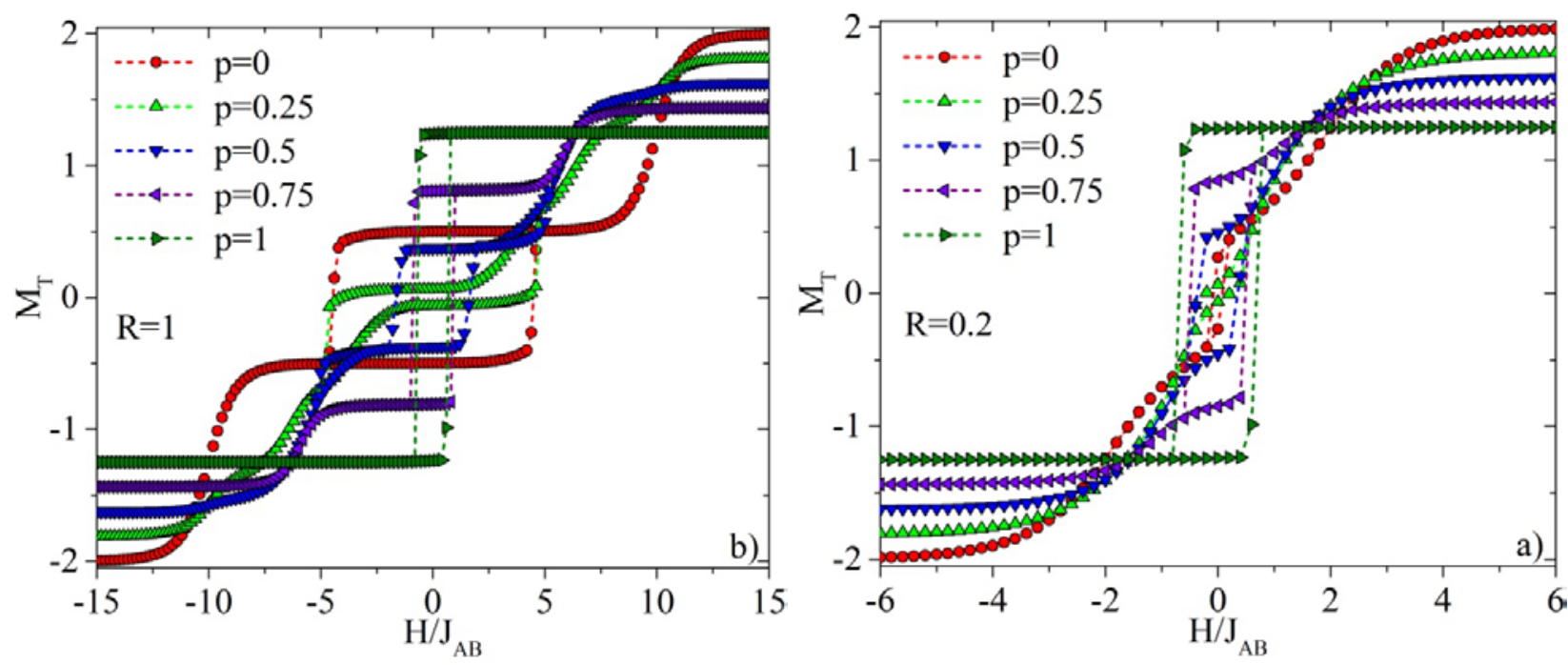

Figure 6: Hysteresis curves of the ternary alloy with a) $R=0.2$, b) $R=1$ for several value of $p$. The curves are shown for reduced temperature value of $k_{B} T_{C} / J_{A B}=1.0$.

As a final investigation, we investigate the temperature dependence of coercivity and remanence of the ternary alloy system at an exchange coupling ratio of $\mathrm{R}=1$ for varying concentration value of type-B atoms in Figure 7(a) and 7(b), respectively. For all concentration values, $H_{c}$ and $M_{r}$ decrease with raising temperature due to the enhanced thermal agitation which destroys the magnetic order in the system and they become zero above the critical temperature of the system. As an example, we show the hysteresis curves of the system for $p=0.5$ and $R=1$ for varying temperature values in Figure 8. The hysteresis loop areas decrease with raising temperature and paramagnetic behavior is observed when the temperature is above the phase transition temperature of the system. 

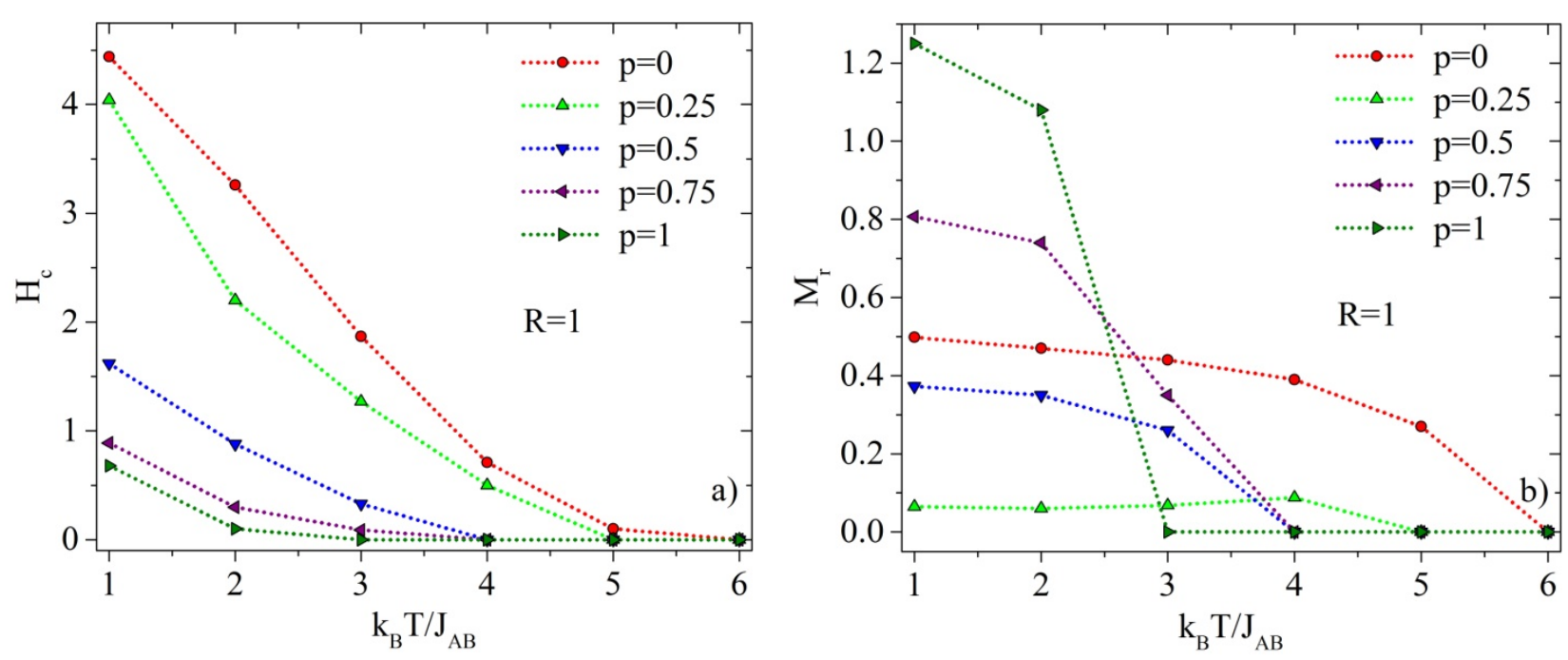

Figure 7: Temperature dependence of the a) coercivity and b) remanence at a exchange coupling ratio of $R=1$ for varying concentration of type-B atoms: $p=0,0.25,0.5,0.75,1$.

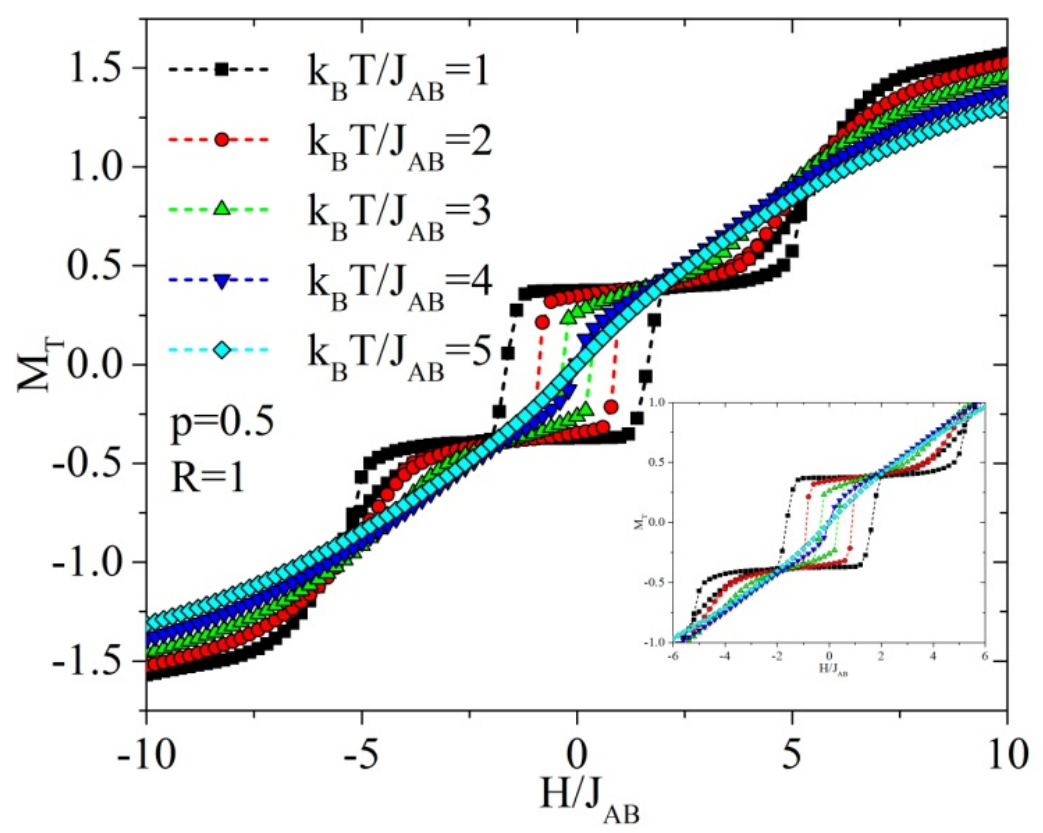

Figure 8: Hysteresis loop of the ternary alloy system for $p=0.5$ and $R=1$ for varying values of the temperature.

As a final remark, we compare our numerical results about the hysteresis features of the ternary alloy system with the literature. From the experimental side, for a ternary metal Prussian blue compound of the type $\left(N i_{p}^{I I} M n_{1-p}^{I I}\right)_{1.5} \mathrm{Cr}^{I I I}(C N)_{6}$, it has been demonstrated that it is possible to modify saturation magnetization, coercivity and remanent magnetization by varying the mixing ratio of p of the system [3]. Therefore, our results are in a qualitative agreement with the available experimental result [3] in literature. On the theoretical side, as far as we know, there are not any works regarding the hysteresis features of ternary alloys. However, the limiting cases of the system $(\mathrm{p}=0$ and $\mathrm{p}=1)$ correspond to mixed Ising spin systems which have been widely studied in literature. For instance, in reference [39], the authours have observed triple hysteresis behaviors similar to our results for a mixed spin-2 and spin5/2 ferrimagnetic Ising system. Also, triple hystresis loops are observed for the other type of mixed spin systems, such as nanowires [40,41], nanoparticles [42,43]. 


\section{CONCLUSIONS}

Hysteresis properties of a mixed ferro-ferrimagnetic ternary alloy of the type $A B_{p} C_{1-p}$ is studied by $\mathrm{MC}$ simulation method with Metropolis algorithm. Both ferromagnetic $\left(J_{A B}>0\right)$ and antiferromagnetic $\left(U_{A C}<0\right)$ spin-spin interactions between nearestneighbor magnetic components are considered. The dependence of the coercivity, remanence and also hysteresis loops of the ternary alloy system on the exchange interaction ratio and concentration value of type-B magnetic components are investigated in detail. Moreover, hysteresis properties of the system are analyzed in a wide temperature region. It has been shown that it is possible to tune the coercivity, remanence and saturation magnetization of the system by changing exchange interaction ratio and number of type-B components. As a further investigation, procedure followed in this work can be applied to nano-dimensional structures such as ternary alloy magnetic nanoparticles and nanowires. Such a study may reveal the effects of reduced dimensions on the magnetic features of ternary alloy systems.

\section{ACKNOWLEDGEMENTS}

The numerical calculations reported in this paper were performed at TÜBITTAK ULAKBIM (Turkish agency), High Performance and Grid Computing Center (TRUBA Resources).

\section{REFERENCES}

[1] J. S. Miller and M. Drillon, Magnetism: Molecules to Materials V, Weinheim: Wiley-VCH, 2005.

[2] S. I. Ohkoshi, O. Sato, T. Iyoda, A. Fujishima, K. Hashimoto, "Tuning of Superexchange Couplings in a Molecule-Based Ferroferrimagnet: $\left(\mathrm{Ni}^{\mathrm{II}}{ }_{\mathrm{x}} \mathrm{Mn}^{\mathrm{II}}{ }_{1-\mathrm{x}}\right)_{1.5}\left[\mathrm{Cr}^{\mathrm{III}}(\mathrm{CN})_{6}\right]$ ”, Inorg. Chem., vol. 36, pp. 268-269, 1997.

[3] S. I. Ohkoshi, O. Sato, T. Iyoda, A. Fujishima, K. Hashimoto, "Magnetic properties of mixed ferro-ferrimagnets composed of Prussian blue analogs”, Phys. Rev. B, vol. 56, pp. 11642, 1997.

[4] S. I. Ohkoshi and K. Hashimoto, "Theoretical treatment of the mixed ferro-ferrimagnets composed of ternary-metal Prussian blue analogs in a paramagnetic region”, Phys. Rev. B, vol. 60, no 18, pp. 12820-12825, June 1999.

[5] S. I. Ohkoshi, S. Yorozu, O. Sato, T. Iyoda and A. Fujishima, "Photoinduced magnetic pole inversion in a ferro-ferrimagnet: (Fe0.40IIMn0.60II)1.5CrIII(CN)6”, Appl. Phys. Lett., vol. 70, pp. 1040, 1997.

[6] S. I. Ohkoshi, Y. Abe, A. Fujishima and K. Hashimoto, "Design and Preparation of a Novel Magnet Exhibiting Two Compensation
Temperatures Based on Molecular Field Theory”, Phys. Rev. Lett., vol. 82, pp. 1285, 1999.

[7] S. I. Ohkoshi, T. Hozumi, M. Utsunomiya, M. Abe and K. Hashimoto, "The observation of two compensation temperatures in a cobalt-manganese hexacyanochromate”, Physica B, vol. 329, pp. 691692, 2003.

[8] S. I. Ohkoshi, T. Hozumi and K. Hashimoto, "Design and preparation of a bulk magnet exhibiting an inverted hysteresis loop”, Phys. Rev. B, vol. 64, pp. 132404, 2001.

[9] P. Bhatt, S. Banerjee, S. Anwar, M. D. Mukadam, S. S. Meena and S. M. Yusuf, "Core-Shell Prussian Blue Analogue Molecular Magnet $\quad \mathrm{Mn}_{1.5} \quad\left[\mathrm{Cr}(\mathrm{CN})_{6}\right] \cdot \mathrm{mH} 2 \mathrm{O} @ \mathrm{Ni}_{1.5}$ $\left[\mathrm{Cr}(\mathrm{CN})_{6}\right] \cdot \mathrm{nH} 2 \mathrm{O}$ for Hydrogen Storage”, Appl. Mater. Interfaces, vol. 6, pp. 17579-17588, 2014.

[10] C-H Lee, C-M Wu, E. Batsaikhan, H-C Li, C. H. Li, M. K. Peprah, D. R. Talham, M. W. Meisel and W-H Li, "Complex Magnetic Phases in Nanosized Core@Shell Prussian Blue Analogue Cubes: $\mathrm{Rb}_{0.48} \mathrm{Co}\left[\mathrm{Fe}(\mathrm{CN})_{6}\right]_{0.75}$ $\left[\left(\mathrm{H}_{2} \mathrm{O}\right)_{6}\right]_{0.25} \cdot 0.34 \mathrm{H}_{2} \mathrm{O} @ \mathrm{~K}_{0.36} \mathrm{Ni}\left[\mathrm{Cr}(\mathrm{CN})_{6}\right]_{0.74}\left[\left(\mathrm{H}_{2} \mathrm{O}\right)_{6}\right]$ $0.26^{\circ} 0.11 \mathrm{H}_{2} \mathrm{O}$ ", J. Phys. Chem. C, vol. 119, pp. 29138-29147, 2015.

[11] P. Bhatt, A. Kumar, S.S. Meena, M.D. Mukadam and S.M. Yusuf, "Magnetic proximity effect in ferrimagnetic-ferromagnetic core-shell Prussian blue analogues molecular magnet”, Chem. Phys. Lett., vol. 651, pp. 155-160, 2016.

[12] P. Zhou, D. Xue, H. Luo and X. Chen, "Fabrication, Structure, and Magnetic Properties of Highly Ordered Prussian Blue Nanowire Arrays", Nano Lett., vol. 2, pp. 845-847, 2002.

[13] P. H. Zhou and D. S. Xue, "Finite-size effect on magnetic properties in Prussian blue nanowire arrays”, J. Appl. Phys., vol. 96, pp. 610-614, 2004.

[14] A. Johansson, E. Widenkvist, J. Lu, M. Boman and U. Jansson, "Fabrication of High-Aspect-Ratio Prussian Blue Nanotubes Using a Porous Alumina Template” Nano Lett., vol. 5, no 8, pp. 1603-1606, 2005.

[15] A. Bobák, F. O. Abubrig and T. Balcerzak, "Multicritical points in the mixed ferromagneticferrimagnetic ternary alloy with a single-ion anisotropy”, Phys. Rev. B, vol. 68, pp. 224405 , December 2003.

[16] A. Bobák, J. Dely and T. Balcerzak, “Compensation temperatures and susceptibility of ternary metal Prussian blue analogs”, Czech. J. Phys, vol: 54, pp. D523-D-526, June 2004.

[17] A. Bobák, F. O. Abubrig and T. Balcerzak, "Phase diagrams of the mixed ferro-ferrimagnetic ternary alloy with a single-ion anisotropy”, J. Magn. Magn. Mater., vol. 272-276, pp. 989-990, 2004.

[18] A. Bobák and J. Dely, “The effect of a singleion anisotropy on the phase diagram of a mixed 
ferro-ferrimagnetic ternary alloy”, Physica A, vol. 341, pp. 281-298, 2004.

[19] J. Dely and A. Bobák, "Magnetic properties of the ternary alloy with a structure of Prussian blue analogs” Physica B, vol. 388, pp. 49-58, 2007.

[20] J. Dely and A. Bobák, "Phase diagrams of the ternary alloy with a single-ion anisotropy in the mean-field approximation”, J. Magn. Magn. Mater., vol. 305, pp. 464-466, 2006.

[21] J. Dely, A. Bobák and D. Horváth , "MeanField and Monte Carlo Study of a Mixed FerroFerrimagnetic Ternary Alloy”, Acta Phys. Pol. A, vol. 113, no 1, pp. 461-464, 2008.

[22] J. Dely, A: Bobák and M. Žukovič, “Critical temperature of a mixed ferro-ferrimagnetic ternary alloy”, J. Phys. Conf. Ser., vol. 200, pp. 022005, 2010.

[23] Bobák, A. O. F. Abubrig and D. Horváth, "Magnetic properties of a mixed ferroferrimagnetic ternary alloy”, Physica A, vol. 312, pp. 187-207, 2002.

[24] H. Hu, Z. Xin and W. Liu, "Magnetic properties of the mixed ferro-ferrimagnets composed of Prussian blue analogs with $\left(\mathrm{A}_{\mathrm{x}} \mathrm{B}_{1-}\right.$ х) $)_{y}$ ”, Phys. Lett. A, vol. 357, pp. 388-392, 2006.

[25]J. Wei, G. H. Yu and L. V. Cheong, "Magnetization of mixed ferri-ferrimagnets composed of Prussian blue analogs $\mathrm{A} 1_{\mathrm{x}} \mathrm{A} 2_{1-\mathrm{x}} \mathrm{B}$ ”, Chin. Phys. B, vol. 20, no 5, pp. 057501, 2011.

[26] E. Albayrak, J. Magn. Magn. Mater., “The mixed-spin ternary-alloy in the form of $\mathrm{AB}_{\mathrm{p}} \mathrm{C}_{1-\mathrm{p}}$ on the Bethe lattice”, vol. 323, pp. 992-996, 2011.

[27]B. Deviren, M. Keskin and Y. Aydin, "Compensation Temperatures, Magnetic Susceptibilities and Phase Diagrams of a Mixed Ferrimagnetic Ternary System on the Bethe Lattice”, JETP Letters, vol. 92, pp. 214-222, 2010.

[28]G. Mert, "Green's function study of a threesublattice mixed-spin Heisenberg ferromagnetic and ferrimagnetic system”, J. Magn. Magn. Mater., vol. 363, pp. 224-231, 2014.

[29] G. M. Buendía and J. E. Villarroel, "Compensation temperatures of mixed ferroferrimagnetic ternary alloys”, J. Magn. Magn. Mater., vol. 310, pp. e495-e497, 2007.

[30]J. Dely, A. Bobák and M. Žukovič, "Compensation temperatures and magnetic susceptibility of a mixed ferro-ferrimagnetic ternary alloy”, Phys. Lett. A, vol. 373, pp. 3197-3200, 2009.

[31] M. Žukovič and A. Bobák, “Critical and compensation phenomena in a mixed-spin ternary alloy: A Monte Carlo study”, J. Magn. Magn. Mater., vol. 322, pp. 2868, 2010.

[32] S. Tsuji, T. Kasama and T. Idogaki, "Decoration-iteration transformation and Monte Carlo simulation for mixed ferrimagnetic ternary alloy”, J. Magn. Magn. Mater., vol. 310, pp. e471e473, 2007.

[33] E. K. Çam and E. Aydiner, J. Magn. Magn. Mater., "Compensation temperature of $3 \mathrm{~d}$ mixed ferro-ferrimagnetic ternary alloy”, vol. 322, pp. 1706-1709, 2010.

[34] E. K. Çam and E. Aydiner, "Magnetic Behavior of Ternary Prussian Blue Analog in Presence Single-Ion Anisotropy”, E. IEEE Trans. Magn., vol. 49, no 9, pp. 4951-4955, 2013.

[35]Y. Yuksel, "Monte Carlo simulation of Prussian blue analogs described by Heisenberg ternary alloy model” J. Phys. Chem. Solids, vol. 86, pp. 207-214, 2015.

[36] E. Vatansever, and Y. Yuksel, "Dynamic phenomena in magnetic ternary alloys”, J. Alloys Compd., vol. 689, pp. 446-450, 2016.

[37] K. Binder, Monte Carlo Methods in Statistical Physics, Berlin: Springer, 1979.

[38] M. E. J. Newman and G. T. Barkema, Monte Carlo Methods in Statistical Physics, Newyork: Oxford University Press, 1999.

[39] W. Wang, D. Lv, F. Zhang, J-L Bi and J-N Chen, "Monte Carlo simulation of magnetic properties of a mixed spin-2 and spin-5/2 ferrimagnetic Ising system in a longitudinal magnetic field”, J. Magn. Magn. Mater., vol. 385, pp. 16-26, 2015.

[40] B. Boughazi, M. Boughrara and M. Kerouad, "The hysteresis behavior of an Ising nanowire with core/shell morphology: Monte Carlo treatment”, J. Magn. Magn. Mater., vol. 363, pp. 26-33, 2014.

[41] W. Jiang, X-X Li, L-M Liu, J-N Chen and F. Zhang, "Hysteresis loop of a cubic nanowire in the presence of the crystal field and the transverse field”, J. Magn. Magn. Mater., vol. 353, pp. 90-98, 2014.

[42] Y. Yuksel, E. Vatansever, and H. Polat , "Dynamic phase transition properties and hysteretic behavior of a ferrimagnetic core-shell nanoparticle in the presence of a time dependent magnetic field", J. Phys.: Condens. Matter , vol. 24, pp. 436004, 2012.

[43] E. Kantar, B. Deviren and M. Keskin, "Magnetic properties of mixed Ising nanoparticles with core-shell structure”, Eur. Phys. J. B, vol. 86, pp. 253, 2013. 